\title{
Exploring Radio Environment Map Architectures for Spectrum Sharing in the Radar Bands
}

\author{
Francisco Paisana *, Zaheer Khan ${ }^{\dagger}$, Janne Lehtomäki ${ }^{\dagger}$, Luiz A. DaSilva ${ }^{*}$, and Risto Vuohtoniemi ${ }^{\dagger}$ \\ * CONNECT, Trinity College Dublin, Ireland \\ Email: \{paisanaf, dasilval\}@tcd.ie \\ $\dagger$ Oulu, Oulu University, Finland \\ Email: $\{$ zaheer, jannel, rv\}@ee.oulu.fi
}

\begin{abstract}
The need for extra spectrum to support future 5G networks and the fact that a large amount of spectrum below $6 \mathrm{GHz}$ is allocated for different radar systems have motivated regulatory bodies and wireless researchers to investigate the feasibility of Dynamic Spectrum Access (DSA) in radar bands. Moreover, the next generation of infrastructures and devices will need to be more flexible to support a much wider range of application services, operate over much wider bandwidths, and exploit multiple radio access technologies. Radio Environment Maps (REMs) can be utilized to enhance the awareness of network entities of their operational radio environment. In the context of potential spectrum sharing in radar bands, REMs can be used to determine spectrum usage and propagation patterns of different incumbent radar systems operating in a given area. This information in turn can be utilized to establish different radar systems protection requirements in a particular band, and can also help to mitigate any interference between incumbent radar systems and Secondary Users (SUs). In this paper, based on spectrum measurement campaigns in Finland and Ireland, we explore the functional architecture of REMs for potential spectrum sharing in radar bands. We argue that the unique operating principle and signal characteristics of this type of incumbent needs to be considered in implementation of the sensor network, REM communication protocol, and REM architecture. We also discuss the potential of REM to facilitate the use of more advanced interference cancellation or avoidance technologies such as temporal sharing that can significantly reduce exclusion zones.
\end{abstract}

Index Terms-Spectrum Sharing, Radar Bands, Radio Environment Map, Temporal Sharing, Sensors.

\section{INTRODUCTION}

Spectrum scarcity has been deemed as one of the main obstacles to the development of new wireless communication technologies [1]. The problem of spectrum scarcity has triggered regulators' interest in novel spectrum sharing mechanisms, which enable coexistence between distinct radio technologies and services.

The opening of TV White Space (TVWS) for wireless communications was one of the first initiatives [2]. As a follow up to the TVWS act, radar bands are now a potential candidate for sharing between wireless communication systems and radar systems. This is due to the fact that radar bands currently occupy a significant portion of the radio spectrum below $6 \mathrm{GHz}$, and these frequencies offer better propagation conditions, and lower costs of semiconductor devices.

Radar systems are used in a wide variety of applications, including avionics, astronomy, mapping, military, weather, and law enforcement. With this variety of uses it is natural that different radar systems require different technologies, different modes of operation, and different interference protection criteria [3], [4]. As a consequence there cannot be one single method to share spectrum with all different radar systems. In contrast, as wireless networks of the future will operate over much wider swathes of spectrum than current systems, it is equally important to design sharing mechanisms that can facilitate unified spectrum selection/aggregation techniques across frequency channels where different types of users operate. One possible solution to this challenge is the use of a Radio Environment Map (REM) based architecture as an enabler to unified spectrum selection/aggregation across different types of spectrum.

The general concept of REM was first introduced by [5]. In [5], [6], REM is defined as a network entity which enhances the awareness of cognitive radios by providing them information about their radio environment. The provided information includes: device locations and their activities, policies and regulation to access spectrum, and other information.

In this paper, we introduce an innovative framework for shared spectrum access in radar bands based on REM. To better understand radar systems' operating principles and to determine their spectrum usage patterns, we first ran an extensive measurement campaign in Finland and Ireland. We measured spectrum usage patterns, and signal characteristics of four types of radar systems, each of which is used for a different application. A more extensive description of the measurements for the particular case of a weather radar system can be found in [7]. Based on the analysed features of each radar system, we propose a unified REM functional architecture that can enhance awareness of network entities with different radio access technologies regarding their radio environment, and can provide support in the channel selection procedure. In contrast to other REM architectures in the literature, our design also facilitates the use of more sophisticated interference mitigation technologies, namely temporal sharing [8], to reduce the effective size of radar systems' exclusion zones. Previously in [8], we proposed several algorithms for estimation of the signal characteristics of radar systems with predictable scan patterns. Contrastingly in this work, we tackle temporal sharing from the REM side, proposing an architecture that also accounts for radar systems that do not display constant rotation periods. 


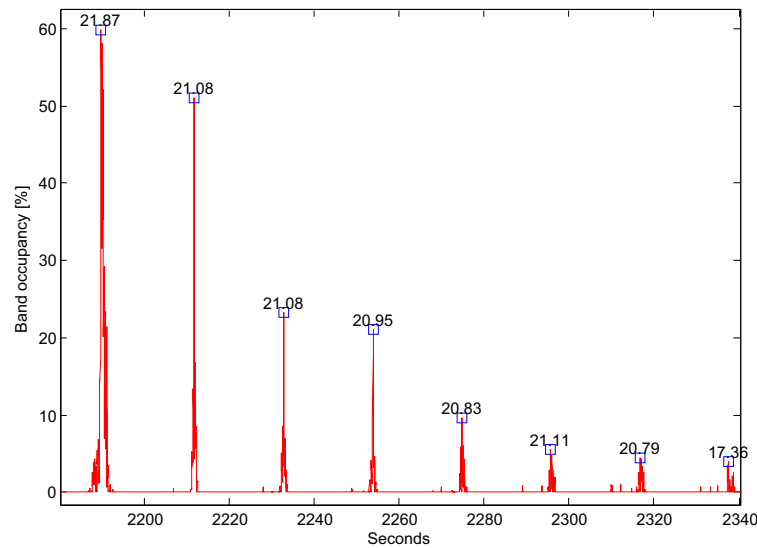

Fig. 1. An example of measured time between main beam peaks of a rotating weather radar operating in the $5 \mathrm{GHz}$ band.

\section{RADAR SySTEMS: BACKGROUND AND SPECTRUM USAGE MODELS}

The use of radio navigation has evolved into a host of technologies that are pushed to the extremes in both civilian and military applications: detecting, ranging, tracking, evading, jamming, and more. Although different radar system characteristics make it difficult to develop a common model to study their spectrum usage, there are radar systems that have basic common features and are also more prevalent than others [4]. For example, in the L, S and C bands, monostatic imaging systems with pulsed waveform are widely used. These systems have co-located transmitters and receivers, and send directional pulses of electromagnetic energy and, through the returning echoes, detect the presence, position, and motion of objects. The emitted Pulse Trains (PTs) can be mainly characterized based on Pulse Repetition Frequency (PRF) or Pulse Repetition Interval (PRI), Pulse Width (PW), Pulse Frequency (PF), and Intra-Pulse Modulation (IPM) [3]. Depending on their application, radar systems can also employ different beam and scanning patterns. The effective range of different radar systems may also vary, usually extending to more than $50 \mathrm{~km}$ [3].

The appealing features of radar spectrum have already led some countries to open parts of the $\mathrm{S}$ and $\mathrm{C}$ bands for wireless broadband services [2]. From the perspective of spectrum sharing with radar systems, existing models of sharing can be grouped into two major types:

- The models that propose sharing only in the spatial dimension, such as the geographic exclusion zone (GEZ) and the Dynamic Frequency Selection (DFS) model.

- The models that along with the space dimension also explore the possibility of sharing in the time dimension. Examples include different zone-based sharing models.

The GEZ and the DFS models both focus on spatial spectrum sharing but using different techniques [1], [9], [10]. The GEZ model utilizes a spectrum management entity called Spectrum Access System (SAS) which manages all secondary users (except the radar incumbents) on the fly, in real time. The SAS's role is to keep every Secondary User (SU) off the incumbent spectrum in the exclusion zones. The exclusion zone is a filled circle centered at the incumbent radar apparatus itself. Several prior works have reported the radii of these exclusion zones to be between 72 and $121 \mathrm{~km}$ [1]. The model may guarantee $100 \%$ protection to the radar systems; however, different works and reports have shown that fixed geographic exclusion zones are unnecessary and counter-productive to the goals of spectrum sharing in the radar bands [1], [11], as they would prevent around $45 \%$ of the US population from spectrum sharing access in the $3550 \mathrm{MHz}$ band. The DFS model also focuses on spatial spectrum sharing with radar systems; however, different from the GEZ model, a DFSenabled device listens and performs processing to detect a radar, and upon detection it moves to another channel and the device is not allowed to scan the channel again for 30 minutes. If a radar is not detected, the device can use the channel but it is still required to periodically scan the channel. This method has two main drawbacks: 1) It is challenging to detect with close to $100 \%$ probability in a way that also minimizes the DFS false alarm rate. As a consequence, different works have reported a DFS-enabled device interfering with a weather radar in the $5 \mathrm{GHz}$ band [1]. 2) DFS is not an efficient mechanism in the search for spectrum opportunities, as it requires long channel availability check time periods, and long non-occupancy periods.

Different from the above two models, zone-based sharing models look for potential sharing opportunities both in the space and the time dimensions. For example, the works in [8], [11] have studied the potential of a zone-based sharing method with rotating radars. Broadly speaking, the zone-based models divide the area around a rotating radar into three zones, where the rotating radar itself is located at the center of the zones. In Zone 1, opportunistic secondary operation is strictly forbidden as it can cause interference on the incumbent radar. In Zone 2, temporal sharing takes place, in which the users can transmit every time the radar's main beam is pointing in another direction. Finally, in Zone 3, the users are free to use the spectrum, as they are outside the interference area of the radar. However, the development of shared spectrum access between radar and communication systems is clearly in its early stages. Many of the precise details of sharing that take into account real spectrum usage of radar systems must still be worked on at this moment [1]. For example, prior works have used theoretical zone-based sharing models that assumed that rotating radars are periodic [11]. However, using real measurements of a weather radar's spectrum usage in the $5 \mathrm{GHz}$ band, our recent work in [7] has shown that weather radar scan patterns are quasi-periodic, and radars can change their scan speed from fast to slow and vice-versa. Moreover, we have also shown that sensing-based methods like DFS may not reliably detect radar signals due to the different vertical scanning angles. The weather radars' rotation period that we measured varies from 13 seconds to 21 seconds. Examples of measured time between main beam peaks of a rotating weather radar operating in the $5 \mathrm{GHz}$ band are shown in Fig. 1. From a temporal sharing perspective, there is a possibility that a considerable amount of spectrum opportunities can be exploited by allowing users inside Zone 2 to transmit when 
the radar antenna's main beam is pointing in another direction.

The results of our work in [7] motivated us to run a measurement campaign to measure spectrum usage behavior of different rotating radar systems in Finland and Ireland. The presented measurement results in [7] include spectrum usage behavior of three ground-based fixed radar systems in Finland: a Weather Radar System in the $5 \mathrm{GHz}$ band, Airport Aircraft Surveillance Radar System in the $1030 \mathrm{MHz}$ uplink channel, 1090 downlink channel, and a surveillance radar in 2200-2245 $\mathrm{MHz}$ channels. The results also include maritime shipborne radar systems in Ireland. From our measurements campaign we have learned the following main lessons:

- Some rotating radars have periodic scan patterns while others have quasi-periodic scan patterns.

- Some systems scan their surroundings in azimuth with a fan-shaped antenna, while, for instance, weather radar systems scan both in azimuth and elevation.

- To avoid co-interference and increase spectrum reuse, radar systems may utilize low duty cycle suppression techniques that filter out signals whose PRI does not match their own. In Fig. 2, we show the measured signal strength when two rotating radar systems placed on Irish Navy ships operate in close vicinity. We were able to deinterleave the two systems' emissions based on their distinct pulse widths.

- Fan-type circular scan radar systems generally have low rotation periods of around 2 seconds, while weather-type radar systems usually have rotation periods higher than 10 seconds.

- Exclusion and temporal sharing zones of a radar system can be dynamic. For example, in the case of a groundbased fixed radar which allows temporal sharing in Zone 2 , the dynamicity of zones can be due to the aggregate interference on the boundary of Zone 1 (exclusion zone). This is because as the density of SUs temporally sharing the radar channel increases, the aggregate interference on the boundary of Zone 1 may also increase which in turn may affect the incumbent located at the center of Zone 1. In such scenarios, it may be required to increase the size of the exclusion zone. In the case of a maritime shipborne radar system, dynamicity of zones can be due to aggregate interference from secondary devices as well as due to mobility of the radar system.

\section{REM DESIGN ASPECTS IN RADAR BANDS}

The design of REM systems raises challenges ranging from scalability to standardization, and security. For the particular scenario of radar spectrum sharing, there are still open questions regarding how such a system would be designed to provide the best trade-off between reliability in spectrum occupancy representation in the spatial, frequency and temporal domains, and deployment costs. Concerns also exist regarding the malicious use of an REM to disclose classified information about military systems to enemy forces, namely their position and waveforms.

We present in this section, some of the technical aspects that we deem more determinant in the implementation of an

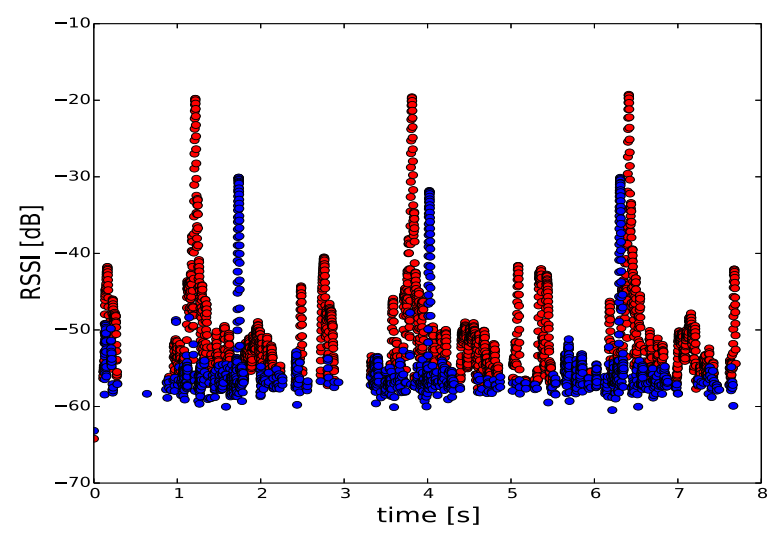

Fig. 2. Measured signal strength when there are two radar systems in the vicinity. Each circle represents a detected pulse with a certain amplitude and time of arrival.

REM and how radar spectrum radio environmental factors may affect them.

\section{A. Number of Measurement Capable Devices (MCDs)}

The number of MCDs is the main contributing factor for the generally high cost associated to the deployment and maintenance of REMs, and places an upper bound on the resolution and reliability of the REM's spatial spectrum occupancy representation. In radar bands, however, we can expect a generally lower density of MCDs needed than in other bands.

The typically long transmit ranges of radar systems, which in many cases require exclusion zones with a radius over 50 $\mathrm{km}$, relax the requirements for high resolution spatial data. Moreover, the heterogeneous deployment of radar stations, normally situated close to coastal areas and to airports, allows a more localized placement of the MCDs in case their position is known a priori.

For the particular case of the $3.5 \mathrm{GHz}$, the US military has raised concerns over the possibility of localization of their ships if the Environmental Sensing Capability (ESC) was ever to be deployed. As a response, and to enforce security requirements, the National Telecommunications and Information Administration (NTIA) proposed that radio environmental data should be obfuscated by placing MCDs sufficiently far from each other along the US coast [12].

The low number and security concerns in military bands make the MCDs deployment likely to take place at a national government level rather than being crowd-sourced. This also reduces the implementation challenges associated to the calibration and identification of untrusted MCDs.

\section{B. REM Update Rate}

The REM update rate defines the level of granularity of spectrum occupancy representation in the time domain. This factor becomes particularly critical for the case of dynamic radio environments, as in such cases the REMs need to be updated frequently, and the resulting spectrum occupancy 
changes need to be propagated to the SUs with the lowest latency possible. This will impose strong requirements on the REM server processing speed, and MCD-server interface channel quality.

In [13], the authors implemented and studied the computational resources and server latency associated to a real-time spectrum monitoring system for the SAS in the $3.5 \mathrm{GHz}$ band. They showed that for a 1 millisecond update rate, the spectrum occupancy server's latency increased significantly for a number of sensor streams higher than 5. On the other hand, the authors also suggested further optimizations that can increase the obtained performance.

The time granularity requirement is interconnected with the type of data the REM intends to extract from radar systems' transmissions. If the goal is to measure the radar system's static exclusion zone size, a rate of one measurement data packet every few seconds per MCD is sufficient. We base this statement on the fact that in this case an MCD should only send data associated to the time instants when it is illuminated by a radar antenna's main beam, which in general corresponds to less than $1 \%$ of the time. On the other hand, if the goal is also to extract data on the radar scan pattern, a more frequent update may be required.

In case the incumbent is placed on mobile platforms, such as in the case of shipborne and airborne systems, the REM may need to be updated every few seconds, depending on the incumbent's speed and conservativeness of the defined exclusion zones.

\section{Centralized versus Distributed Cognitive Engine}

The received IQ samples by an individual MCD may go through several stages of processing, compression and aggregation, before arriving to a central REM. These stages can take place at the MCDs level, where statistics of the received samples are obtained, and/or in localized small-scale REMs that can perform data fusion from many different sources before forwarding the resulting data to the central REM.

To determine the exclusion zone size of a radar system, the MCD can perform some prior analysis of the sensed data, selecting the samples relative to what is perceived as an illumination of the MCD by a radar antenna main lobe. These samples are then fused with the ones from other nearby MCDs at a local REM to obtain more reliable estimates on spectrum occupancy in all domains.

\section{Algorithmic Complexity}

The methods employed in the processing of the MCD measurement data and construction of the REM may have different levels of complexity depending on the required accuracy demands, and the need to discriminate between different types of transmitters, in particular, whether they had origin in an incumbent or SU. This can be done at the MCD level through the employment of feature detection/estimation or matched filter algorithms, or at the REM level through analysis of the signal strength variations over time, frequency, and space. Moreover, propagation model interpolation methods may need to take to account changes caused by the radar mobility.

\section{REM BASED ARCHITECTURE FOR SPECTRUM SHARING}

In this section, we present a general REM-based architecture for spectrum sharing with different radar systems. The proposed architecture can be divided into three components: 1) An REM repository; 2) Different radar operators; 3) Network entities, such as dedicated MCDs, user devices, and access points (APs) or Base Stations (BSs). The REM repository is a collection of resources that can be accessed by network infrastructures, such as APs/BSs, to retrieve information about their radio environment. The REM repository consists of: 1) an Information and Measurement Resource Module (IMRM); 2) a database module (DM); and 3) a spectrum manager (SM).

In Fig. 3, we illustrate an example scenario for the proposed REM architecture. In the considered scenario there is a wireless network located in an area that has in its vicinity four different radar systems' sites. The four radar systems are labeled as A, B, C and D, where the radar systems A, B, and $\mathrm{C}$ are ground-based fixed radar systems, and $\mathrm{D}$ is a mobile radar system mounted on a ship near the coast. Fig. 3 also illustrates simplified high level block diagrams for different components involved in the proposed REM-based spectrum sharing architecture. Next we explain the elements of the proposed architecture.

\section{A. The REM repository elements}

1) Information and Measurement Resource Module (IMRM): The IMRM collects low-overhead spectrum usage information from different radar operators, and sensing reports from the deployed MCDs.

- Input from radar systems: In the case of fixed groundbased rotating radars, the static information includes: 1) location of a radar system; 2) scan pattern type and rotation period; 3) transmit power and antenna maximum gain, and beamwidth; 4) maximum INR level.

The dynamic information includes: 1) Any scheduled change in rotation speed of radar systems that display quasi-periodic scan patterns, such as weather systems operating in the $5 \mathrm{GHz}$ band. 2) In the case of civil radar systems placed in mobile platforms, the REM can receive notification updates on their location from their navigation databases directly.

We highlight that, for the case of most civil radar systems, the information on their position and spectrum usage is open and can be accessed through several websites. For the case of the weather radar that we measured, its alterations in rotation speed are also known and are scheduled several hours in advance. These factors enable the implementation of the proposed cooperative REM, with minimal changes to the primary systems.

- Input from MCD network: The MCDs collect information on the radar signal strength, time of arrival, and waveform features. As most radar signals consist of pulse trains with very high peak power and low duty cycle, we envision the use of sensing algorithms based on peak detection or autocorrelation analysis, as the ones proposed in [8]. The detected pulses are then clustered based on their waveform features and time of arrival, each cluster 


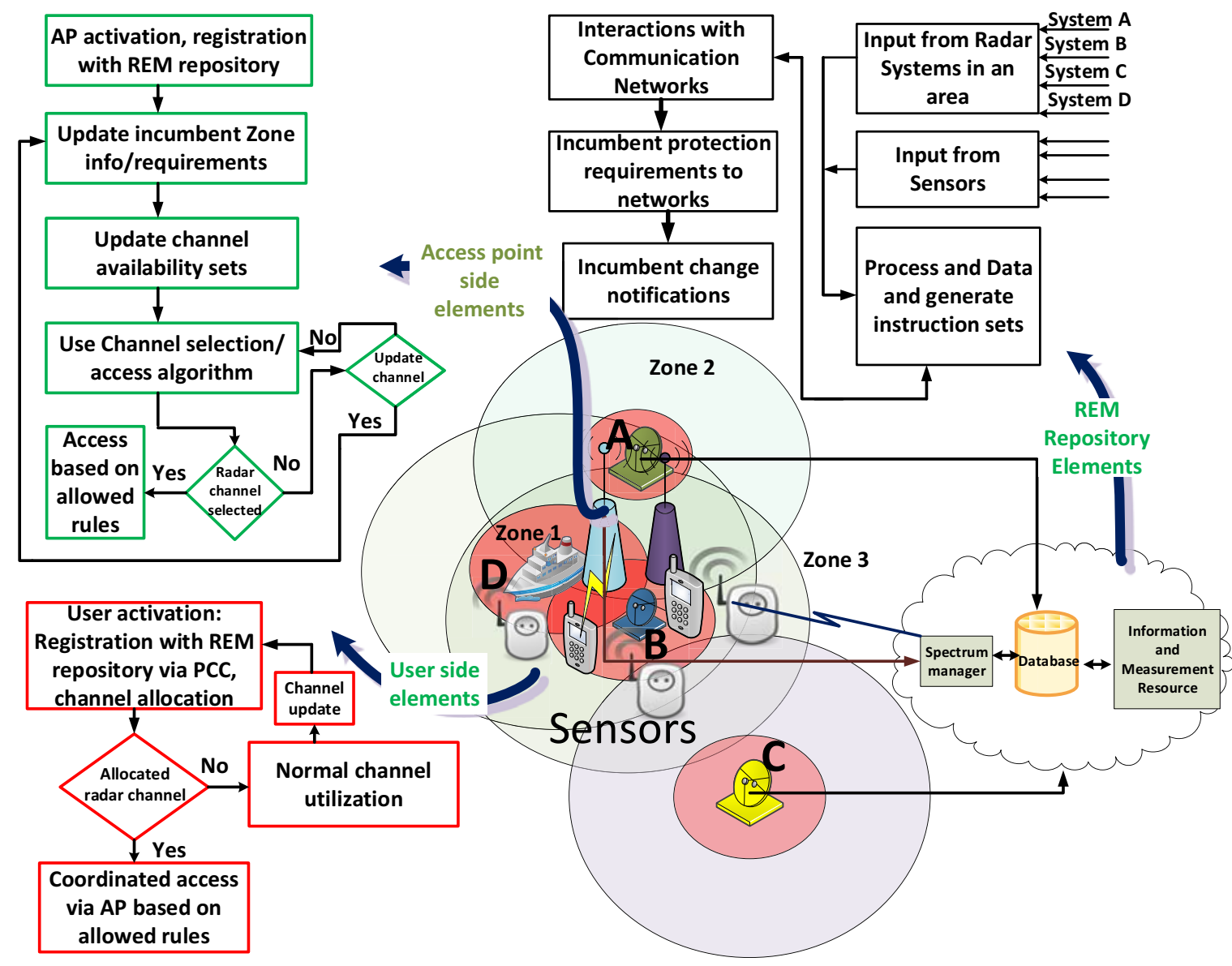

Fig. 3. An example scenario for the proposed REM architecture and also simplified high level block diagrams for different components involved in the proposed REM-based spectrum sharing architecture.

being efficiently represented through a set of description words, such as average amplitude, pulse width, and centre frequency. The rate at which measurement data is sent to the REM may be quite low, only consisting of the clusters' description word sets taken at the instants of time MCDs are illuminated by radar antenna lobes.

2) Database module (DM): The DM processes the information from the IMRM and generates instruction sets for different network entities operating in the area. In particular, the DM may list the channels that are available in each area for sharing, and for each channel, the rules for its access, in particular, whether more advanced access schemes like temporal sharing are allowed.

At an initial stage, the input from radar operators alone should be sufficient for the DM to dimension exclusion zones based on conservative theoretical propagation models. These zones can then get more refined as more signal strength measurements become available from the MCDs. As the MCDs' reports may also provide data regarding the time of arrival and waveform features of the detected signals, the DM can use this data to deinterleave transmissions from different radar systems and SUs, without the use of expensive highly specialized sensing algorithms at the MCDs.
Deintearleaving of different systems' emissions has special relevance in mobile incumbent scenarios, as it reduces location ambiguity, allows the update of each system's exclusion zone independently, and facilitates temporal sharing with multiple Primary Users (PUs). It also provides some insight on the spectrum usage by the SU networks, and in particular, the existence of any malicious or malfunctioning device transmitting in forbidden channels.

For the case of systems placed on mobile platforms, the uncertainty regarding their exclusion zones caused by the latency associated with the incumbent location and MCD measurement updates can be circumvented through spatial error regions.

For the case of military bands, where secrecy of the systems' position is crucial for their operation, the DM may obfuscate the IMRM data by extending incumbent exclusion zone sizes, before notifying APs/BSs about the list of available channels in a given area at a given time. Moreover, the DM instruction set provides a secure way of ensuring sharing with such radar systems as the access is controlled and managed by a trustworthy authority which is authorized to operate in a given area by an official regulatory body. 
3) Spectrum Manager (SM): A spectrum manager (SM) on one side interacts with communication network entities, such as APs or BSs, to collect their location information, and transmission power characteristics, and on the other side collects generated instructions from the DM. It then combines this data and notifies the network entities about which portions of spectrum are available to them for sharing. When a radar channel is selected by an AP or a BS then the SM module provides rules of sharing for the selected channel, and radar scan related notifications when temporal sharing is allowed. The SM module also notifies the need for SUs to move to another channel when incumbent protection requirements are violated due to, for instance, aggregated interference.

\section{B. The Access Point/Base station elements}

An SU AP/BS in order to get channel access authorization needs first to register in the SM module. This procedure can be carried out through an internet connection. The registration of an AP involves providing its location information, and transmission power characteristics in order for the list of available/forbidden channels to be computed.

The AP in return obtains a list of available channels, and rules for sharing. The SU updates its list of channels, which is now a set of licensed/unlicensed channels and available radar channels, and using a standard unified spectrum access algorithm the SU selects a channel. If the selected channel is a radar channel, its access is based on the rules provided by the SM module, otherwise normal mode of access is utilized. When a radar channel is selected the SM module needs to notify any changes in radar's operation, such as scan change notifications, if any. When the AP must vacate the channel due to its own QoS requirements and/or due to the incumbents protection requirements, the AP repeats the mentioned process of channel list update and rules for sharing before attempting to access a new channel.

\section{The User elements}

As opposed to infrastructure entities, such as APs/BSs, user devices can be mobile, which adds extra complexity in terms of protecting the radar incumbents. For instance, it may be challenging to detect when a mobile user crosses an exclusion zone and notify it to vacate the incumbent channel in a timely fashion. Bootstrapping challenges may also arise regarding which wireless channels are available for the user to communicate with the REM. Due to these issues, it is more viable that mobile devices only access radar channels when associated to an AP/BS operating in licensed bands, as in this case, the control signaling has a more predictable QoS, and the users' geographic locations are more easily tracked.

Under the proposed REM model, the steps taken by a user device to register in the SM and select a channel in which to operate should be equivalent to the ones followed by APs/BSs. When using a radar channel, the user should also maintain its connection to the REM alive to receive notifications on possible changes in radar spectrum usage patterns, and vacate its current channel if needed.

\section{Conclusions}

The main contribution of this paper is the proposal and design of REM-enabled shared spectrum access between radar and wireless communications systems. It explains how lowoverhead information signals from different radar systems and the deployed sensor network in a given area are collected, and processed in an REM repository. It also explains the mechanism which allows the transformation of processed REM information to be utilized by the secondary users for unified channel access in licensed and radar bands.

In our future work, we will use analytical and simulation based results to assess the overhead of REM collection/processing and dissemination in the network.

\section{ACKNOWLEDGMENTS}

This material is based upon works supported by the Science Foundation Ireland under grants no. 10/CE/I1853 and 10/IN.1/I3007. This work was also funded by Academy of Finland under the grant number 268997.

\section{REFERENCES}

[1] Institute for Telecommunication Sciences, "Developing forward thinking rules and processes to fully exploit spectrum resources: Case study exploring approaches for real-time federal spectrum sharing," in ISART 2012 Proceedings, NTIA Special Publication SP-14-508, 2014.

[2] FCC, "Enabling Innovative Small Cell Use In 3.5 GHz Band NPRM \& Order," Docket 12-148, 2012.

[3] "Agilent radar measurements," Application Note, Agilent Technologies, 2014

[4] H. Griffiths, L. Cohen, S. Watts, E. Mokole, C. Baker, M. Wicks, and S. Blunt, "Radar Spectrum Engineering and Management: Technical and Regulatory Issues," Proceedings of the IEEE, vol. 103, no. 1, pp. 85$102,2015$.

[5] Y. Zhao, "Enabling cognitive radios through radio environment maps," Ph.D. dissertation, Virginia Tech, USA, 2007.

[6] Z. Wei, Q. Zhang, Z. Feng, W. Li, and T. Gulliver, "On the construction of radio environment maps for cognitive radio networks," IEEE Wireless Communications and Networking Conference (WCNC), 2013.

[7] Z. Khan, J. J. Lehtomaki, R. Vuohtoniemi, E. Hossain, and L. A. DaSilva, "On opportunistic spectrum access in radar bands: Lessons learned from measurement of weather radar signals," to appear in Wireless Communications Magazine, 2016.

[8] F. Paisana, J. P. Miranda, N. Marchetti, and L. A. DaSilva, "Databaseaided sensing for radar bands," in IEEE International Symposium on Dynamic Spectrum Access Networks (DYSPAN), 2014, pp. 1-6.

[9] F. Paisana, N. Marchetti, and L. A. DaSilva, "Radar, TV and Cellular Bands: Which Spectrum Access Techniques for Which Bands?" IEEE Communications Surveys and Tutorials, vol. 16, no. 3, pp. 1193-1220, 2014.

[10] ITU-R, "Dynamic frequency selection in wireless access systems including radio local area networks for the purpose of protecting the radiodetermination service in the $5 \mathrm{ghz}$ band," Recommendation M.1652$1,2011$.

[11] M. Tercero, K. W. Sung, and J. Zander, "Temporal Secondary Access Opportunities for WLAN in Radar Bands," in 14th International Symposium on Wireless Personal Multimedia Communications (WPMC), 2011, pp. $1-5$.

[12] E. Drocella, J. Richards, R. Sole, F. Najmy, A. Lundy, and P. Mckenna, "3.5 GHz Exclusion Zone Analyses and Methodology," Tech. Rep., 2015

[13] M. Souryal, M. Ranganathan, J. Mink, and N. E. Ouni, "Real-time centralized spectrum monitoring: Feasibility, architecture, and latency," in 2015 IEEE International Symposium on Dynamic Spectrum Access Networks (DySPAN), 2015, pp. 106-112. 